\title{
The effect of the "rod-and-frame" illusion on grip planning in a sequential object manipulation task
}

\author{
Céline Crajé · John van der Kamp • \\ Bert Steenbergen
}

Received: 9 March 2007/ Accepted: 5 September 2007/Published online: 2 October 2007

(C) Springer-Verlag 2007

\begin{abstract}
We investigated the effect of visual context (i.e., a visual illusion) on the planning of a sequential object manipulation task. Participants $(n=13)$ had to grasp a rod embedded in a "rod-and-frame" illusion and insert the rod-end into a tight hole in a pre-defined way. The grip type (defined by start posture, either pronated or supinated; and end posture, either comfortable or uncomfortable) used to grasp the rod was registered as a macroscopic variable of motor planning. Different rod orientations forced the participants to switch between grip types. As expected, most participants switched between pronated and supinated start postures, such that they ended the movement with a comfortable end posture. As it has been argued that planning is dependent on visual context information, we hypothesized that the visual illusion would affect the specific rod orientation at which participants would switch into a different grip type. This hypothesis was confirmed. More specifically, the illusion affected the critical spatial information that is used for action planning. Collectively, these findings are the first to show an effect of an illusion on motor planning in a sequential object manipulation task.
\end{abstract}

C. Crajé $(\bowtie) \cdot$ B. Steenbergen

Nijmegen Institute for Cognition and Information (NICI),

Radboud University Nijmegen, Montessorilaan 3,

6525 HR Nijmegen, The Netherlands

e-mail: c.craje@nici.ru.nl

J. van der Kamp

Faculty of Human Movement Sciences, VU University,

Amsterdam, The Netherlands

J. van der Kamp

Institute of Human Performance, University of Hong Kong,

Pok Fu Lam Road, Hong Kong
Keywords Motor planning - Visual illusion · Perceptual judgment · Grip selection

\section{Introduction}

At present, there is a lively debate about the effects of visual illusions on the planning and control of discrete grasping actions (for reviews, see Glover 2004; Carey 2001). An influential model in this respect is the perception-action model of Milner and Goodale (1995; Goodale and Milner 1992, 2004). The perception-action model posits a dissociation between two functionally and structurally different visual pathways in the brain: a ventral stream processing "vision for perception" (the "what-system") and a dorsal stream processing "vision for action" (the "how-system"). The ventral pathway processes information used for the conscious recognition and identification of objects. The dorsal pathway, however, has the purpose to guide goal directed actions, and is therefore dependent on information about the spatial properties and coordinates of an object with respect to the actor. Since these different pathways depend on different sources of visual information (i.e., context dependent information for the ventral stream and context independent information for the dorsal stream), the perception-action model predicts that a visual illusion will affect perception, but not action, a prediction for which ample evidence exists (e.g., Aglioti et al. 1995; Haffenden and Goodale 1998; Dyde and Milner 2002).

Recently, Glover proposed the planning-control model (Glover 2002; Glover and Dixon 2001a, 2001b, 2002), in which a dissociation between visual representations that subserve planning and those that are used for on-line control of action is postulated. This model posits that representations responsible for planning entail a broad 
range of current visual and cognitive information about (1) spatial (e.g., size, shape, orientation) and non-spatial (e.g., function, weight, fragility) properties of the target object, (2) the overarching goal of the action, and (3) the visual context surrounding the target. This information is integrated with knowledge from past experience (Glover 2004, p. 4). Representations responsible for on-line control, however, are solely aimed at minimizing the spatial error of the movement and are focused on the spatial characteristics of the target object. Support for the planningcontrol model has been found in experiments investigating the effects of a visual illusion on action. As an example, Glover and Dixon (2001b) had participants grasp a bar placed in front of a grated background in such a way that an orientation illusion was induced. The bar could be grasped with an overhand grip or an underhand grip. The results showed that grip choice was affected by the visual background. Hence, these findings indicate that the selection, or planning, of a particular grip type is subject to the visual context surrounding the target. In a second experiment, using the same experimental set-up, Glover and Dixon investigated planning and control by measuring kinematic parameters during transport of the hand to the bar. Contrary to the first experiment, participants were not free in their grip choice, and had to use the same, predefined grip during the whole session. In line with the predictions of the planning-control model, hand orientation was affected by the illusion in the initial part of the movement, but this effect decreased when the hand approached the bar. Thus, initial planning, as evidenced by grip type and initial kinematic parameters of the reach, was affected by the illusion, but during transport of the arm the kinematic parameterization was corrected.

Although studies that investigated the impact of the visual context surrounding the target object on the planning and control of action are abundant (e.g, Aglioti et al. 1995; Jackson and Shaw 2000; Danckert et al. 2002; Franz 2001; Franz et al. 2005; Mendoza et al. 2006; Van Doorn et al. 2007), the evidence is restricted to simple prehension tasks that did not demand any further action with the grasped object: the goal of the action was to merely to grasp and lift the object. No studies have yet investigated the effect of visual context in situations where the overarching goal of the action does not coincide with simply grasping and lifting the object. This is surprising, since tasks such as a sequential object manipulation task, in which a target is grasped for a specific purpose, provide a method 'par excellence' to evaluate planning processes. By using a sequential object manipulation task, it can be assessed whether planning processes also take the visual context surrounding of an object into account when the overarching goal of the action requires further manipulation of the grasped object.
A distinctive feature of sequential object manipulation tasks is that they require anticipatory planning, i.e., the forthcoming perceptual-motor demands associated with the goal of the action sequence need to be taken into account when initially grasping an object (cf., Johnson-Frey et al. 2004). In other words, the type of grasp is not only determined by the characteristics of the target object and its visual surrounding, but must also accommodate the ensuing task requirements. Ample evidence for such anticipatory planning can be found in the studies of Rosenbaum and co-workers. They showed that the selection of a particular grip type is indicative for motor planning. Participants preferred to grasp an object with a grip type that enabled them to end the task in a comfortable posture, the so-called 'end-state comfort' effect (Rosenbaum and Jorgensen 1992; Rosenbaum et al. 1992, 1993, 1996; Cohen and Rosenbaum 2004; Short and Cauraugh 1999). Stated differently, participants sacrifice initial comfort for the sake of final comfort, implying anticipatory motor planning. For example, in the Rosenbaum and Jorgensen study (1992) participants had to grasp and rotate a bar that was mounted on a clock-face. Positions on the clock-face were separated by $45^{\circ}$, and participants were instructed to take hold of the bar and rotate it to a pre-defined target position. The results showed that participants adapted their initial posture, such that it enabled them to end the task in a comfortable posture. Obviously, to attain this comfortable end posture, they switched between overhand and underhand grip types when grasping the bar (for similar findings from a different theoretical background, see Kelso et al. 1994). Summing up, the studies of Rosenbaum et al. showed that initial grip type is indicative for motor planning in a sequential object manipulation task, and second, they showed that initial comfort is sacrificed to attain posture comfort at the end of the task. Additionally, previous studies have shown similar effects for kinematic parameterization. For example, Marteniuk et al. (1987, see also Gentilucci et al. 1997) showed that the goal of the second movement was reflected in movement kinematics of the first movement. Likewise, Steenbergen et al. (1995) showed that the strength of joint couplings of the first movement was critically dependent on the task constraints of the second movement.

At present, no study has scrutinized the effects of visual context on movement planning in a sequential task that requires anticipatory planning. Earlier studies on the effects of visual context on the planning of action used simple grasping movements. For instance, in the experiments of Glover and co-workers the action goal was to grasp the bar, no further manipulation was required. Hence, the posture with which the bar was grasped was identical to the posture at the end of the task. Consequently, no conflict occurred between comfort of the initial posture and comfort of the 
end posture. In a sequential task however, the initial posture with which an object is grasped and the posture at the end of the task are not necessarily the same because additional movements are made after grasping the object. Therefore, when planning the initial posture in a sequential object manipulation task, participants have to take the constraints arising from the end posture into account as well, i.e., they are engaged in anticipatory planning (Johnson-Frey et al. 2004).

In the present study, the effect of visual context on anticipatory planning of a sequential object manipulation task was investigated. Specifically, participants had to grasp a rod embedded in a "rod-and-frame" illusion (i.e., a rod surrounded by a tilted frame) and subsequently place the rod-end vertically in a tight hole. Both, rod and frame could be independently rotated. Following Rosenbaum et al. (1992) we expected adaptations in the initial posture such that a comfortable end posture is reached. Specifically, participants are expected to switch between pronated and supinated initial postures at a specific rod orientation. Based on the planning-control model (Glover 2002, 2004), in which context effects are not distinguished with respect to the different components of planning, it is hypothesized that the exact rod orientation at which this switch occurs is affected by the rotation of the frame.

\section{Method}

\section{Participants}

Thirteen right-handed college students (three males, ten females), aged 18-27 years (mean age 22.6 year/month, SD 2.10 year/month) participated in the experiment for money or course credit (see Table 1 for participant information). All participants had normal or corrected-tonormal vision, were naïve to the purpose of the experiment, and had no known neurological deficits. This study was approved by the local ethics committee and performed in accordance with the ethical standards laid down in the 1964 Declaration of Helsinki.

\section{Experimental set-up and apparatus}

The participants were comfortably seated in a chair positioned in front of a table upon which the experimental setup was placed (see Fig. 1). The stimulus consisted of a white 3D "rod-and-frame" illusion that was placed in front of a black curtain $(220 \times 105 \mathrm{~cm})$. This curtain was used to prevent any visual cues of veridical frames of reference, such as the ceiling or the floor. Both the rod (length: $15 \mathrm{~cm}$, diameter: $3.5 \mathrm{~cm}$ ) and the surrounding frame
$(30 \times 30 \times 2.3 \mathrm{~cm})$ could be rotated independently such that the rod-and-frame illusion was created. The rod had a grey marker on one side signifying the end that had to be placed upwards in the hole (diameter: $5 \mathrm{~cm}$ ) of a box. After the participant had placed the rod in the hole, an experimenter sitting next to the participant replaced the rod to the set-up and scored the used grip type. Rotation of rod and frame was performed manually by a second experimenter who sat behind the curtain. Participants wore liquid crystal occlusion goggles to prevent them seeing the rotation of the frame and rod in-between trials. The goggles could be switched from opaque to transparent in less than $30 \mathrm{~ms}$.

\section{Procedure}

The study consisted of two experimental sessions that were conducted in succession. First, an action task was performed, second we performed a perception task to assess participants' perceptual sensitivity for the illusion [these tasks are denoted as (1) Action task and (2) Perception task in what follows]. Standard rest breaks were present between sessions, and on participants' demands.

\section{Action task}

The action task consisted of a pre-measurement and the main experiment. The procedure for both was as follows. A trial started when the participant pressed the button on the button-box with the index finger of the preferred (right) hand. Subsequently, the goggles were closed and the second experimenter manually changed the rod and frame orientation. When ready (i.e., within $2 \mathrm{~s}$ ) the goggles opened, which was the start-signal for participants to grasp the rod as quickly as possible and place it vertically with the marker facing upwards in a hole of a tight fitting box that was located in front of them, slightly to the right of the body midline. Participants were asked to grasp the rod with a power grip, i.e., with the thumb on one side of the rod and the fingers on the other side. Once the rod was grasped, participants were not allowed to change the grip type during rotation of the rod. This was necessary, because it urged participants to plan the task prior to grasping the rod. If this had not been the case and participants were allowed to manipulate the rod in-hand, then it would not have been strictly necessary for participants to plan the movement prior to grasping the rod.

As dependent variable, the grip type that participants used to grasp the rod was measured. The grip types were evaluated on two criteria: the start posture of the hand and the end posture of the hand. The start posture was scored as either a "pronated" (overhand) or a "supinated" (underhand) 
Table 1 Participant information

\begin{tabular}{lllllll}
\hline Part & M/F & Age (year) & Percep & Pro: supi & comf:uncomf & Strategy \\
\hline 1. & F & 23 & 0.90 & $100: 125$ & $225: 0$ & Comfortable ender \\
2. & F & 22 & 0.92 & $24: 201$ & $225: 0$ & Comfortable ender \\
3. & F & 25 & 0.97 & $101: 124$ & $223: 2$ & Comfortable ender \\
4. & F & 20 & 0.82 & $108: 117$ & $225: 0$ & Comfortable ender \\
5. & F & 21 & $\times$ & $52: 173$ & $224: 1$ & Comfortable ender \\
6. & M & 25 & 0.90 & $82: 143$ & $222: 3$ & Comfortable ender \\
7. & F & 27 & 0.79 & $87: 138$ & $225: 0$ & Comfortable ender \\
8. & F & 20 & 0.90 & $123: 102$ & $225: 0$ & Comfortable ender \\
9. & M & 26 & 0.80 & $146: 79$ & $225: 0$ & Comfortable ender \\
10. & M & 27 & $\times$ & $138: 87$ & $225: 0$ & Comfortable ender \\
11. & F & 18 & 0.90 & $225: 0$ & $38: 187$ & Pronation starter \\
12. & F & 19 & 0.74 & $225: 0$ & $224: 1$ & Pronation starter \\
13. & F & 19 & 0.70 & $56: 169$ & Pronation starter \\
\hline
\end{tabular}

Part: participant number; M/F male/female; age: age in years; Percep: Perception task—percentage correct answers; Pro:supi: Start posture— number of pronated start postures: number of supinated start postures; Comf:uncomf: End posture—number of comfortable end postures: number of uncomfortable end postures; Strategy: strategy used in the action task (see text for description)

a
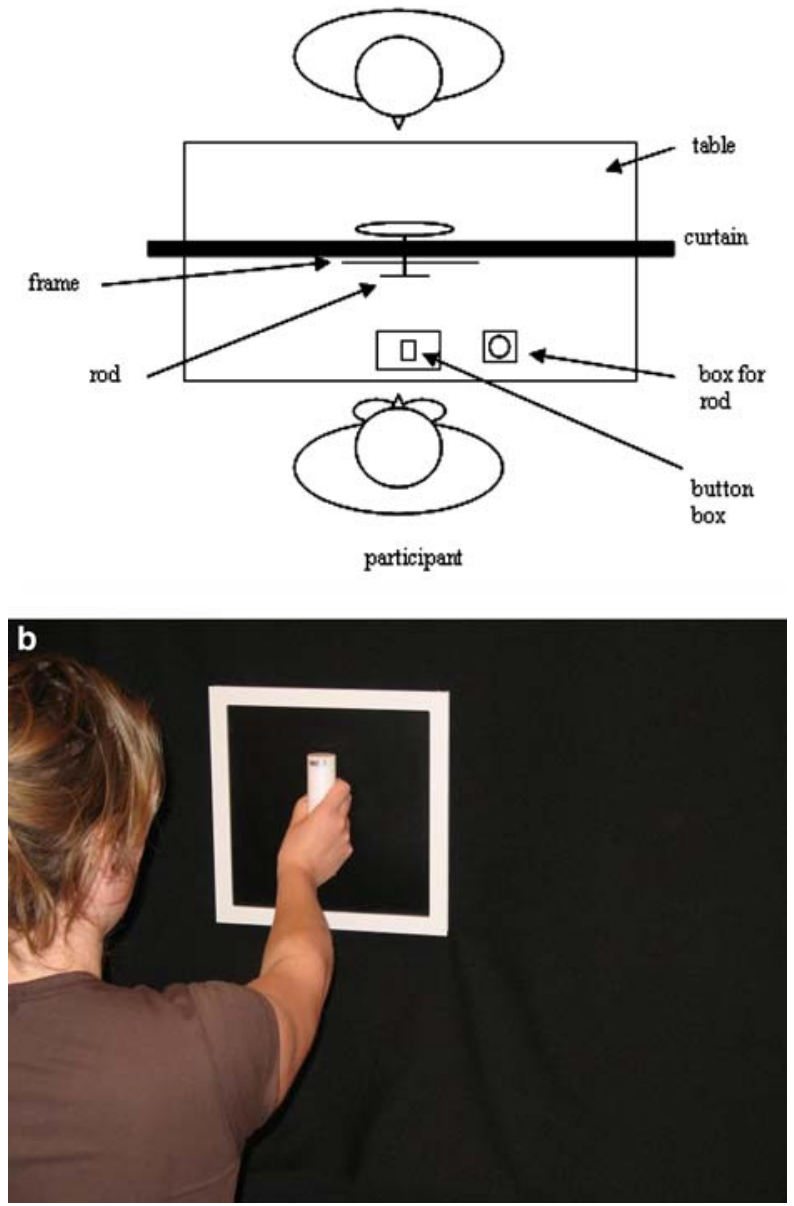

Fig. 1 Schematic drawing of the experimental setup, viewed from above (a) and a photograph of the first author grasping the rod (b) posture. The end postures were scored as "comfortable" when a grip with the thumb towards the marker was used and as "uncomfortable" when a grip with the thumb away from the marker was used (see also, Steenbergen et al. 2000; Rosenbaum and Jorgensen 1992). Since the start and end posture are not fully independent of each other, the combination of start and end posture was labeled as the grip type. Three different grip types were distinguished: grip type 1, a pronated initial posture resulting in a comfortable end posture, grip type 2, a supinated initial posture resulting in a comfortable end posture and grip type 3 , a pronated initial posture resulting in an uncomfortable end posture (see Fig. 2). The combination of a supinated initial posture resulting in an uncomfortable end posture was theoretically possible. However, this combination was never used, and will therefore not be mentioned in what follows.

With respect to the grip type, our primary interest was the rod orientation at which a switch into another grip type occurred. The rod orientation at which there was an equal chance to observe both grip types was denoted the "switch point".

\section{Pre-measurement}

As the location of the switch point differed between individuals, we performed a pre-measurement prior to the main experiment. In this pre-measurement the individual switch point of each participant was established. In general, switches in grip types occur in the lower half of the "clock face" (e.g., Rosenbaum et al. 1992; Steenbergen et al. 2000), but individual differences are present as to the exact 
Fig. 2 The grip type scoring system used to establish the grip type that participants used. Grip types were defined by the combination of the initial posture (pronated or supinated) and the end posture (comfortable or uncomfortable). Explanation, see text

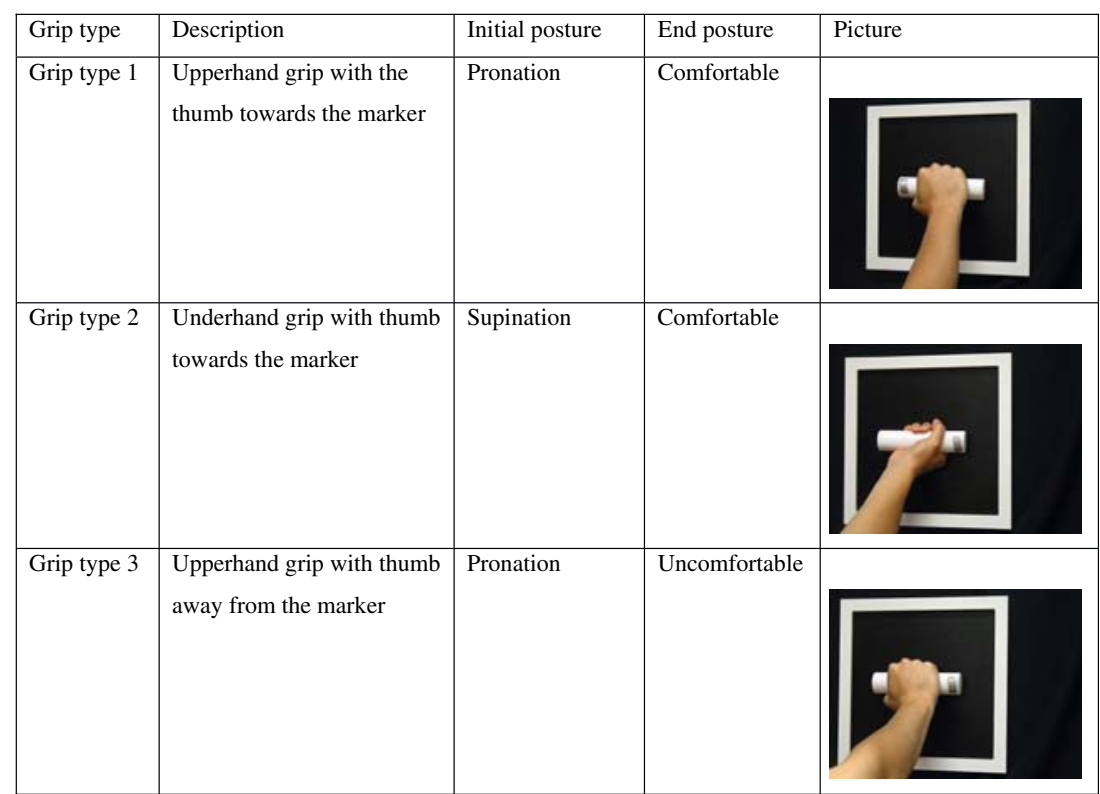

orientation of the rod where the switch occurs. During the pre-measurement the frame was not rotated. Rod orientations were presented in a range of $180^{\circ}$, from the horizontal rod orientation with the marker on the left side (denoted as $90^{\circ}$ ), via the vertical rod orientation with the marker facing downwards (denoted as $0^{\circ}$ ) to the horizontal rod orientation with the marker on the right side (denoted as $90^{\circ}$ ). Thirteen rod orientations were tested, separated by equal angles of $15^{\circ}$ (see Fig. 3). Every rod orientation was presented three times in a completely randomized order, resulting in a total of 39 trials. The switch point was determined by the rod orientation where participants switched between two different grip types, thus, at this rod orientation there was an equal chance to observe both grip types. For most participants the switch point was restricted to one rod orientation. When the grasping pattern consisted of a range of rod orientations, the mathematical middle of that range was taken to be the switch point for that participant. The pre-measurement took approximately $15 \mathrm{~min}$.

\section{The main experiment}

The rod orientations during the main experiment were normalized to the individual switch points, which allowed us to study the individual switch region into detail without overloading participants with too many trials. Measurements were performed in a range of $80^{\circ}$ surrounding the individual switch point, separated by angles of $10^{\circ}$. This resulted in a total of nine rod orientations that were tested in the main experiment $\left(-40^{\circ},-30^{\circ},-20^{\circ},-10^{\circ}, 0^{\circ}, 10^{\circ}, 20^{\circ}, 30^{\circ}, 40^{\circ}\right.$ relative to individual switch point). Negative orientations are clockwise rod orientations compared with the individual switch point, whereas positive orientations are directed

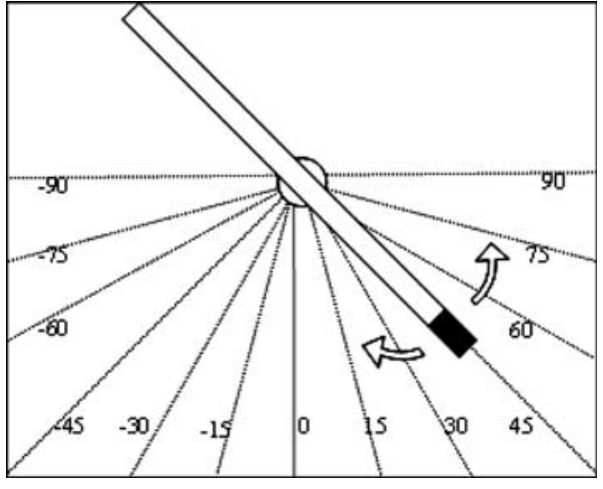

Fig. 3 Schematic drawing of the 13 rod positions used in the premeasurement of the action task. In this figure the rod is oriented at $45^{\circ}$. The black side of the rod represents the marker. Note that the color coding is inconsistent with the experiment where we used a black background, whereas the rod and the surrounding frame were colored white (see Figs. 1b, 2 )

counterclockwise to the switch point. During the experiment we also manipulated the orientation angle of the frame, such that the "rod-and-frame" illusion was created. The frame was rotated in either a clockwise $(\mathrm{CW})$ or a counterclockwise $(\mathrm{CCW})$ direction. A total of five frame orientations were used $\left(20^{\circ} \mathrm{CCW}, 10^{\circ} \mathrm{CCW}, 0^{\circ}, 10^{\circ} \mathrm{CW}, 20^{\circ} \mathrm{CW}\right)$ yielding a total of 45 unique conditions. In each condition, five trials were performed in a completely randomized order. The main experiment, involving 225 trials, took about $45 \mathrm{~min}$ for each participant.

\section{Perception task}

We performed a perception task to assess participants' perceptual sensitivity for the illusion. It was examined whether different rotations of the surrounding frame 
affected the perceived orientation of the rod. To that end, two rod-and-frame combinations were sequentially shown to the participant. First, a rod surrounded by a tilted frame was shown, followed by either the same or a different oriented rod surrounded by Frame $0^{\circ}$. In between presentations, the goggles were closed for less than $2 \mathrm{~s}$. Participants had to report if the orientation of the rod was the same or different in the two displays. In the majority of the trials the rod orientation did not change between presentations (for example, when the first display was a combination of Rod $-30^{\circ}$ and Frame $20^{\circ} \mathrm{CCW}$, the second display combined Rod $-30^{\circ}$ with Frame $0^{\circ}$ ). In this perception task, 4 frame rotations $\times 9$ rod orientations $\times 3$ repetitions were tested, yielding 108 trials. In addition, we also added 72 "catch trials" (4 Frame rotations $\times 9 \operatorname{rod}$ orientations $\times 2$ directions of rod changes), where the rod orientation actually did change between the two presentations, either $10^{\circ} \mathrm{CW}$ or $10^{\circ} \mathrm{CCW}$. The main reason to add catch trials was to prevent that participants could anticipate that the two rods were the same in all trials. However, catch trials were not used in the analyses. The total of 180 trials was presented in a completely randomized order. The perception-task took about $45 \mathrm{~min}$ to be carried out.

Data analysis

\section{Action task}

Analysis of pilot recordings revealed that participants used two strategies to perform the action task. Although all participants used grip type 1 in some of the trials, at the individual switch point differences in grip type choice appeared. While most of the participants switched to an underhand initial posture resulting in a comfortable end posture (grip type 2), some participants switched to an overhand initial posture resulting in an uncomfortable end posture (grip type 3). Consequently, two movement strategies could be delineated. One group of participants switched between grip type 1 and grip type 2 and always ended with a comfortable end posture (this strategy is denoted as "comfortable enders"), whereas the other group of participants switched between grip type 1 and grip type 3 and always started with a pronated initial posture (this strategy is denoted as "pronation starters").

At the individual switch point every participant used grip type 1 in approximately $50 \%$ of the trials, irrespective of the strategy employed, because participants either switched between grip type 1 and grip type 2 (comfortable enders) or between grip type 1 and grip type 3 (pronation starters). This allowed us to collapse the data and to use the same scoring method for both strategies, that is, the frequency of grip type 1. For every participant individually, logistic (S-shaped) functions were fitted through the mean frequency of grip type 1, separately for the five different frame orientations and on the basis of a least squares fitting method (see Van Doorn et al. 2007 for a similar method). The function was of the form where $y$ is the assigned score, i.e., the location of the switch point, $x$ is the rod orientation, $c$ is the rod orientation of the switch point and $k$ is a measure of the slope at that point

$y=1 / 1+\mathrm{e}^{-k(x-c)}$.

Using this method, for every participant the location of the switch point (i.e., the rod orientation where a participant switched between grip types) was determined for the five frame orientations. In order to calculate the illusion effect, the value of the switch point in the control condition $\left(0^{\circ}\right.$ frame rotation) was subtracted from the value of the switch point in the experimental conditions (where the frame was rotated). As our prime interest was the effect of visual context on planning, rather than the direction of the illusion effect, we used absolute difference scores. Moreover, the direction of the illusion effect was not similar among participants, a finding that is not uncommon in the "rodand-frame" illusion literature (e.g., Beh and Wenderoth 1971; DiLorenz and Rock 1982). The absolute difference scores were analyzed using a repeated measures Analysis of Variance (ANOVA) with frame as within subjects factor.

\section{Perception task}

The number of errors per condition were analyzed using a 4 (frame: $\left.20^{\circ} \mathrm{CCW}, 10^{\circ} \mathrm{CCW}, 10^{\circ} \mathrm{CW}, 20^{\circ} \mathrm{CW}\right) \times 5($ rod: $-20^{\circ},-10^{\circ}, 0^{\circ}, 10^{\circ}, 20^{\circ}$ ) repeated measures ANOVA. Frame as a factor in the ANOVA denoted the first frame that is presented to the participant. The second frame was always the same, i.e., $0^{\circ}$.

\section{Results}

Action task

During the experiment, participants showed the same grasping behavior as in the pre-measurement phase, that is, they switched between different grips at a particular rod orientation. The average switch point over all conditions was at rod orientation $-3^{\circ}$ (for the "comfortable enders" at rod orientation $-6^{\circ}$ and for the "pronation starters" at rod orientation $6^{\circ}$ ), all were in the lower half of the clock face. For the negative rod orientations (i.e., rod orientations that are rotated clockwise compared with the individual switch 
point) participants used grip type 1 , which is an overhand initial posture resulting in a comfortable end posture. Conversely, at the positive rod orientations (i.e., rod orientations that are rotated counterclockwise compared with the individual switch point) the grip patterns were less consistent. Most participants $(n=10)$ switched to an underhand initial posture leading to a comfortable end posture, while some $(n=3)$ switched to an overhand initial posture leading to an uncomfortable end posture ("comfortable enders" and "pronation starters", respectively, see also Table 1).

To answer our main research question (does visual context affect anticipatory planning?), we analyzed the effect of frame orientation on the location of the switch point. For each participant individually, we calculated at which rod orientation they switched between grips for all frame orientations, using a logistic function. This way, we could calculate the magnitude (in degrees) by which the switch point had shifted in the experimental conditions compared with the control condition. In Fig. 4 the data of four participants are shown (participants 9, 10, 12 and 13). In the figure, the different frame orientations are depicted on the $x$-axis (with Frame $0^{\circ}$ as the control condition), whereas the $y$-axis represents the location (i.e., rod orientation) of the switch point. It can be derived that the location of the switch point is different in the control condition and the experimental conditions. However, the effect of frame was not in the same direction for all participants. Therefore, absolute different scores between the switch point of the control condition (i.e., Frame $0^{\circ}$ ) and the switch point in the four experimental conditions (i.e., Frame $10^{\circ} \mathrm{CW}$, Frame $20^{\circ}$, Frame $10^{\circ} \mathrm{CCW}$ and Frame $20^{\circ} \mathrm{CCW}$ ) were calculated as a measure of the illusion effect. The mean absolute illusion effect (i.e., the amount of degrees that the switch point had shifted compared with the control condition) was $5.3^{\circ}$ for Frame $20^{\circ} \mathrm{CCW}, 9.3^{\circ}$ for Frame $10^{\circ} \mathrm{CCW}, 7.4^{\circ}$ for Frame $10^{\circ} \mathrm{CW}$ and $6.0^{\circ}$ for Frame $20^{\circ} \mathrm{CW}$ (see Fig. 5). A repeated measures ANOVA revealed a significant effect of Frame $[F(4,48)=3.29$, $P<0.05$ with Greenhouse Geisser correction for sphericity]. Pairwise comparisons showed that the illusion effect of Frame $20^{\circ} \mathrm{CCW}$, Frame $10^{\circ} \mathrm{CCW}$ and Frame $20^{\circ} \mathrm{CW}$ were significantly different from the control condition (all $P<0.05)$.

\section{Perception task}

Data of two participants (participants 5 and 10, see Table 1) were not used for analyses due to technical problems. The mean score of all participants was 0.85 (SD 0.084 ), indicating that in $85 \%$ of the trials participants correctly reported that the perceived rod orientation in the

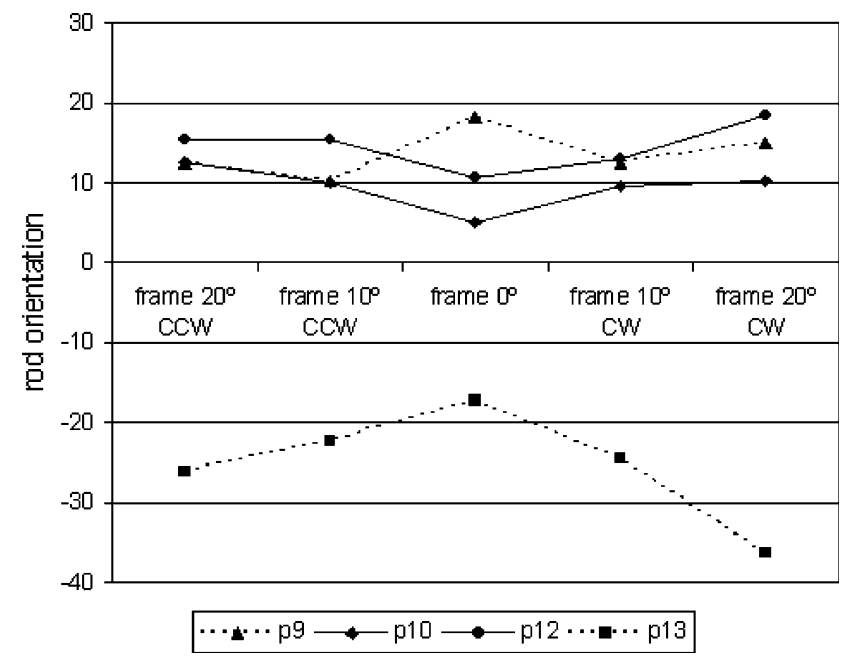

Fig. 4 Location of the switch point (i.e., rod orientation) in the five frame rotation conditions in four participants $(9,10,12,13)$. On the $x$ axis the five frame orientations are depicted, whereas the $y$-axis represents the rod orientation of the switch point

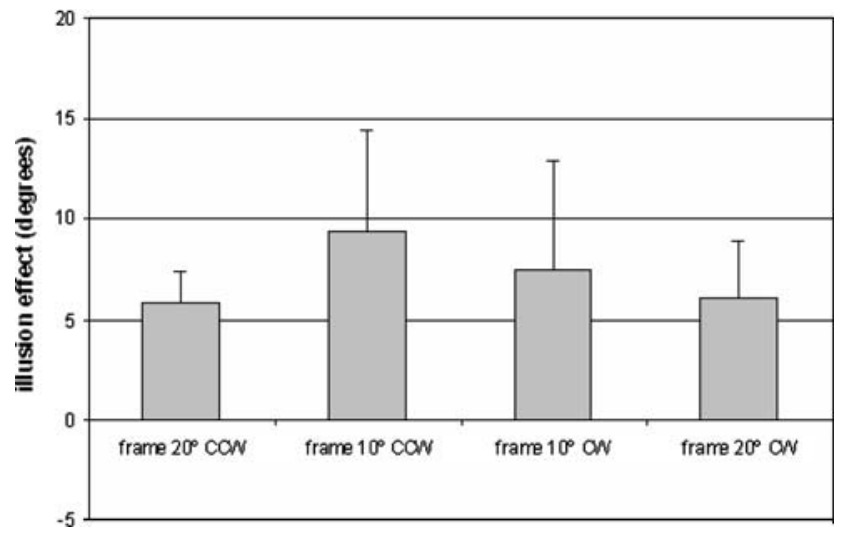

Fig. 5 The absolute effect of frame rotation on the switch point for the four frame orientations (averaged over all participants). Error bars indicate $2 \mathrm{SE}$ of the mean. The absolute effects are calculated by subtracting the value of the switch point in the control condition from the experimental conditions, hence, the control condition is not depicted here. On the $x$-axis the different frame orientations are plotted, whereas the $y$-axis represents the magnitude (in degrees) by which the switch point was shifted compared with the control condition

two presentations was not different. The percentages of correct answers varied between $70 \%$ (participant 13) and 97\% (participant 3). The mean score in the 'comfortable end posture' group was $87 \%$ compared with $78 \%$ in the "pronation start posture" group. However, this between subjects effect of strategy just failed to reach significance $[F(1,9)=3.74, P=0.085]$. A repeated measures ANOVA on the total number of errors revealed a significant effect of Frame $[F(3,27)=4.14, P<0.05]$. The percentages of correct answers were $86 \%$ for Frame $20^{\circ} \mathrm{CCW}, 90 \%$ for Frame $10^{\circ} \mathrm{CCW}, 87 \%$ for Frame $10^{\circ} \mathrm{CW}$ and $77 \%$ for 
Frame $20^{\circ} \mathrm{CW}$. Post hoc comparisons showed significant differences between Frame $20^{\circ} \mathrm{CW}$ and Frame $10^{\circ} \mathrm{CW}$ $(P=0.051)$ and between Frame $20^{\circ} \mathrm{CW}$ and Frame $10^{\circ}$ CCW $(P<0.05)$.

\section{Discussion}

The purpose of the work reported here was to evaluate the influence of visual context on the planning of a sequential object manipulation task. Earlier research on the effects of visual illusions on action was limited to simply grasping a target object without any further purpose. By contrast, in the present study we asked participants to grasp a target object to subsequently place it in a hole. This task requires anticipatory planning, in which constraints arising from the end posture prevail in initial grip choice. That is, the initial grip must accommodate the upcoming movements. As far as we know, no other study has scrutinized visual context effects in such a sequential, object manipulation task. In our study, a rod was embedded in a typical "rod-andframe" illusion configuration. We used a wide range of rod orientations that would force participants to switch between different grip types if they were to reach a comfortable posture at the end of the task. The effect of visual context on anticipatory planning processes was investigated by measuring if the location of the switch point shifted when the surrounding frame was tilted.

The main finding of our study was that the frame manipulations affected the location of the switch point (i.e., the rod orientation where participants switched between grip types), and thus the motor planning of the initial grip type towards the target object. Although earlier findings have already shown that the kinematics and joint couplings in the first movement towards a target object are affected by the upcoming second movement (Gentilucci et al. 1997; Marteniuk et al. 1987; Steenbergen et al. 1995), our results extend these finding by showing that initial grip planning is also affected by the visual context. However, in line with earlier findings on the illusion effects of the "rod-and-frame illusion" (e.g., Beh and Wenderoth 1971; DiLorenz and Rock 1982) the results did not show a consistent direction of the illusion effect among participants. This phenomenon is due to the complex interaction between the specific location of the individual switch point with the frame orientation and the individual sensitivity for the illusion.

Our results are in line with at least three contemporary models that make specific predictions about the effect of illusions on action (planning). First, following the predictions of Glovers' model (2004) we hypothesized that the visual context would affect the specific rod orientation at which participants switch to a different grip. This hypothesis was confirmed as the location of the switch point was affected by the surrounding frame. These findings extend observations of Glover and Dixon (2001b; Glover et al. 2005; see also Van Doorn et al. 2007), in which an orientation illusion was shown to affect grip choice in a simple grasping task. Second, our findings are in line with the predictions stemming from the perceptionaction model (Milner and Goodale 1995). Goodale and Milner propose that the ventral stream is responsible for "the perceptual representation of the perceptual world that is used in the planning of actions" (Goodale and Milner 2004, p. 38), thus assuming action planning to be subject to a visual illusion. Support for the hypothesis that the ventral stream plays an important role in action planning has also been reported in a patient study by Dijkerman et al. (2003), in which two patients with ventral stream lesions did not show appropriate switching when grasping bars in different orientations. Finally, our results can also be accommodated by the common-representation model of Franz (2001), in which it is proposed that a visual illusion affects both perception and action. It is important to note here that our study was not aimed at providing a critical test for one of these models. Rather, we aimed to examine what components of planning are affected by visual context.

The "rod-and-frame" illusion has been investigated by Dyde and Milner (2002), who found that the illusion influenced perception but not action. At first glance, these results may appear contradictory to our findings, but we argue that the difference in task constraints may have contributed to the different findings. As Smeets et al. (2002) argued, different tasks necessitate different types of spatial information to be used for action. For example in the Dyde and Milner study (2002) participants grasped the ends of the rod between their thumb and forefinger and participants were therefore dependent on the visual information regarding the position of the ends of the rod. In contrast, the orientation of the rod constituted the relevant action-related information source for participants in our study. Smeets and Brenner (1995) and Smeets et al. (2002) have proposed that an illusion only effects on action when the critical spatial characteristics of the target in the relation to the to-be-performed action are affected by the illusion. In our study this critical spatial characteristic was the orientation of the rod, whereas in the Dyde and Milner study it was the position of the ends of the rod.

Finally, two issues should be mentioned here, namely, the comparison between the perception task and the action task, and second, the unexpected finding of two strategy groups. The first issue concerns the comparison of illusion effects on perception and action. As Franz (2001) pointed out, an inherent problem in visual illusion studies is the comparison between the perception and the action task, as these tasks are predominantly measured by different methods, as was also the case in the present experiment. 
However, although our study does not allow us to compare perception and action in a quantitative way, the perception task did provide information about how participants perceived the rod orientation when surrounded by a tilted frame. Specifically, participants' perception of the rod orientation was affected by the surrounding frame.

The second issue concerns the finding of two strategies. Our results showed that the means (i.e., grip type) by which the end goal was reached was affected by the visual illusion, however, participants reached the end goal differently, viz used different strategies. Most participants switched between pronated and supinated start postures, such that they ended the movement with a comfortable end posture. Still, three participants in our study did not obey this "end-posture comfort" rule. They used a pronated start posture that resulted in both uncomfortable and comfortable end postures. Importantly, however, irrespective of the strategy used, the effect of the visual context on grip planning was consistent. That is, tilting the frame affected the rod orientation where participants switched their grip, but depending on the strategy most participants switched between grip type 1 and 2 and some participants switched between grip type 1 and 3 . This unexpected finding begs the question as to why some participants used a strategy that did not enable them to end the task in a comfortable end posture? The "posture based motion planning"-model of Rosenbaum et al. (2001) assumes that prior to movement execution an end posture is chosen from the stored posture base. The model further assumes a time constraint for this search process. If enough time is allowed, the search will most likely result in a posture that satisfies the end comfort criterium. If, however, insufficient time is allowed for the search, end postures may be selected that are not optimal. More specifically, these postures may be uncomfortable or even unfit for the task (see Meulenbroek et al. 2001 for model simulation and validation). In our study we instructed participants to perform the task "as fast as possible". Therefore, it may be speculated that the "pronation starters" have put more emphasis on the speed of responding, thereby not completely searching their stored posture base. As the group of "pronation-starters" was small $(n=3)$, we cannot draw any definite conclusions on this matter but further examination is warranted.

Acknowledgments The first author was supported by a grant from the Netherlands Organization for Scientific Research (NWO, grant number 400-04-046).

\section{References}

Aglioti S, DeSouza JFX, Goodale MA (1995) Size-contrast illusions deceive the eye but not the hand. Curr Biol 5(6):679-685
Beh HC, Wenderoth PM (1971) The effect of variation of frame shape on the angular function of the rod-and-frame illusion. Percept Psychophys 11:35-37

Carey DP (2001) Do action systems resist visual illusions? Trends Cogn Sci 5(3):109-113

Cohen RG, Rosenbaum DA (2004) Where grasps are made reveals how grasps are planned: generation and recall of motor plans. Exp Brain Res 157:486-495

Danckert JA, Sharif N, Haffenden AM, Schiff KC, Goodale MA (2002) A temporal analysis of grasping in the Ebbinghaus illusion: planning versus online control. Exp Brain Res 144:275-280

Dijkerman HC, Schindler RD, McIntosh RD, Nijboer TCW, Milner AD (2003) Choosing between alternative wrist postures: action planning needs perception. Congress of European Neuropsychological Societies, Modena, Italy

DiLorenzo JR, Rock I (1982) The rod-and-frame effect as a function of the righting of the frame. J Exp Psychol Human 8(4):536-546

Dyde RT, Milner AD (2002) Two illusions of perceived orientations: one fools all of the people some of the time; the other fools all of the people all of the time. Exp Brain Res 144:518-527

Franz VH (2001) Action does not resist visual illusions. Trends Cogn Sci 5(11):457-459

Franz VH, Scharnowski F, Gegenfurntner KR (2005) Illusion effects on grasping are temporally constant not dynamic. J Exp Psychol Human 313(6):1359-1378

Gentilucci M, Negrotti A, Gangitano M (1997) Planning an action. Exp Brain Res 115:116-128

Glover S, Dixon P (2001a) The role of vision in the on-line correction of illusion effects on action. Can J Exp Psychol 55(2):96-103

Glover SR, Dixon P (2001b) Dynamic illusion effects in a reaching task: evidence for separate visual representations in the planning and control of reaching. J Exp Psychol Human 27(3):560-572

Glover S (2002) Visual illusions affect planning but not control. Trends Cogn Sci 6(7):288-292

Glover S, Dixon P (2002) Semantics affect the planning but not control of grasping. Exp Brain Res 146:383-387

Glover S (2004) Separate visual representations in the planning and control of action. Behav Brain Sci 27:3-78

Glover S, Dixon P, Castiello U, Rushworth MFS (2005) Effects of an orientation illusion on motor performance and motor imagery. Exp Brain Res 166:17-22

Goodale MA, Milner AD (1992) Separate visual pathways for perception and action. Trends Cogn Sci 15(1):20-25

Goodale MA, Milner AD (2004) Plans for action. Behav Brain Sci 27(1):37-40

Haffenden AM, Goodale MA (1998) The effect of pictorial illusion on prehension and perception. J Cogn Neurosci 10(1):122-136

Jackson SR, Shaw A (2000) The ponzo illusion affects grip-force but not grip-aperture scaling during prehension movements. J Exp Psychol Human 26:418-423

Johnson-Frey SH, McCartney ME, Keen R (2004) Reaching beyond spatial perception: effects of intended future actions on visually guided prehension. Vis Cogn 11(2/3):371-399

Kelso JAS, Buchanan JJ, Murata T (1994) Multifunctionality and switching in the coordination dynamics of reaching and grasping. Hum Mov Sci 13:63-94

Marteniuk RG, MacKenzie CL, Jeannerod M (1987) Constraints on Human arm movement trajectories. Can J Psychol 41:365-378

Mendoza JE, Elliott D, Meegan DV, Lyons JL, Welsh TN (2006) The effect of the Müller-Lyer Illusion on the planning and control of manual aiming movements. J Exp Psychol Hum Percept Perform 32(2):413-422

Meulenbroek RGJ, Rosenbaum DA, Jansen C, Vaughan J, Vogt S (2001) Multijoint grasping movements Simulated and observed effects of object location, object size, and initial aperture. Exp Brain Res 138:219-234 
Milner AD, Goodale MA (1995) The visual brain in action. Oxford University Press, New York

Rosenbaum DA, Jorgensen MJ (1992) Planning macroscopic aspects of manual control. Hum Mov Sci 11:61-69

Rosenbaum DA, Vaughan J, Barnes HJ, Jorgensen MJ (1992) Time course of movement planning: selection of handgrips for object manipulation. J Exp Psychol Learn 18(5):1058-1073

Rosenbaum DA, Vaughan J, Jorgensen MJ, Barnes HJ, Steward E (1993) Plans for object manipulation. In: Myer DE, Kornblum S (eds) Attention and performance, vol 14. MIT, Cambridge, pp 803-820

Rosenbaum DA, van Heugten CM, Caldwell GE (1996) From cognition to biomechanics and back: The en-state comfort effect and the middle-is-faster effect. Acta Psychol 94:59-85

Rosenbaum DA, Meulenbroek RJ, Vaughan J, Jansen C (2001) Posture-based motion planning: applications to grasping. Psychol Rev 108(4):709-734
Short MW, Cauraugh JH (1999) Precision and the end-state comfort effect. Acta Psychol 100:243-252

Smeets JBJ, Brenner E (1995) Perception and action are based on the same visual information: distinction between position and velocity. J Exp Psychol Hum 21(1):19-31

Smeets JBJ, Brenner E, de Grave DDJ, Cuijpers RH (2002) Illusions in action: consequences of inconsistent processing of spatial attributes. Exp Brain Res 147:135-144

Steenbergen B, Marteniuk RG, Kalbfleisch LE (1995). Achieving coordination in prehension: Joint freezing and postural contributions. J Motor Behav 27:333-348

Steenbergen B, Hulstijn W, Dortmans S (2000) Constraints on grip selection in cerebral palsy. Minimising discomfort Exp Brain Res 134:385-397

Van Doorn H, Van der Kamp J, Savelsbergh GJP (2007) Grasping the Müller-Lyer illusion: the contributions of vision for perception in action. Neuropsychologia 45(8):1939-1947 